\title{
Quality of Life of Patients with Cardiac Pacemaker: Levels, Associated Characteristics, and the Impact of Anxiety and Depression
}

\author{
Maria Polikandrioti \\ University of West Attica, Athens, Greece \\ Corresponding author: Maria Polikandrioti, University of West Attica, Athens, Greece; E-mail: mpolik2006@yahoo.com
}

Received: 20 Jan 2021 Accepted: 15 Apr 2021 Published: 28 Feb 2022

Citation: Polikandrioti M. Quality of life of patients with cardiac pacemaker: levels, associated characteristics, and the impact of anxiety and depression. Folia Med (Plovdiv) 2022;64(1):117-27. doi: 10.3897/folmed.64.e63234.

\begin{abstract}
Introduction: Implantation of a permanent cardiac pacemaker (PPM) improves recipients' quality of life (QoL). However, psychiatric disturbance may adversely affect QoL and undermine clinical outcomes.
\end{abstract}

Aim: To explore impact of anxiety and depression on QoL of PPM recipients.

Materials and methods: A hundred and fifty PPM recipients were enrolled. Data collection was performed by completing the Hospital Anxiety and Depression Scale (HADS) and the SF-36 Health Survey (SF-36). Demographic characteristics were also included. The statistical significance level was $p<0.05$.

Results: Almost a quarter of the patients experienced anxiety (25.3\%) and depression (26.0\%). In terms of QoL, patients showed moderate to high levels in social functionality, energy/fatigue, emotional well-being and pain (median: 50, 60, 72, and 67.5, respectively) and poor levels in physical functioning, physical role and emotional role (medians: 22, 0 , and 0 respectively). In addition, patients had moderate levels of general health (median 48.5). All QoL subscales were negatively associated with anxiety and depression of patients on a univariate level $(p<0.05)$. Regarding demographic variables affecting QoL, age was statistically significantly associated with physical role $(p=0.025)$, emotional role ( $p=0.005)$, social functioning $(p=0.033)$, and pain $(p=0.018)$. Furthermore, physical role was statistically significantly associated with number of children $(p=0.024)$, emotional role with education level $(p=0.011)$, social functioning with family status $(p=0.018)$, and general health with residency $(p=0.006)$.

Conclusions: Demographic characteristics and anxiety/depression are related with QoL. A better understanding of these associations may help clinicians in planning rational and cost-effective interventions.

\section{Keywords}

anxiety, depression, quality of life, HADS, SF36, pacemaker

\section{INTRODUCTION}

During recent decades, implantation rates of permanent cardiac pacemakers (PPMs) have increased dramatically mainly due to improvements in diagnosis of cardiac diseas$\mathrm{es}^{1,2}$ and progress in pacing technology. ${ }^{3}$

According to estimates, over one million PPMs are implanted globally every year ${ }^{4}$, out of which 250000 are im- planted in the United States alone. ${ }^{5}$ In 2011, average implantation rate in Europe was 938 devices per million inhabitants while in Greece, it was 629 per million inhabitants. ${ }^{3}$

Implantation rates are increasing markedly with advancing age. More in detail, $70 \%-80 \%$ of all PPMs are implanted in individuals aged more than 65 years while incidence rates for men exceed those for women. ${ }^{6}$ Demand of pacing therapy is anticipated to double within 25 years in accor- 
dance with population aging and differing etiologies of cardiac arrhythmias. ${ }^{6}$

This electronic device improves patients' quality of life (QoL) up to one year post implantation. ${ }^{7,8}$ QoL reflects patients' perspective ${ }^{7,9}$ thus providing information alongside with the traditional physiologic measurements of health status. ${ }^{10}$ Furthermore, QoL is not only a key element to achieve patient-centred approach but also stimulates clinicians' awareness of patient concerns. Moreover, QoL is acknowledged as a valuable tool to recognize effectiveness of pacing therapy on patients' daily functioning. ${ }^{8}$ Needless to say, QoL has become more valued than extending life under limited or disabled conditions. ${ }^{7}$

According to guidelines, monitoring of recipients is suggested in periods between 3 and 12 months post implantation. ${ }^{9}$ Each follow up visit includes assessment of device function and cardiovascular events, as well as evaluation of patients' physical status. ${ }^{9}$ Therefore, the time of scheduled follow-up is the most suitable to explore QoL. ${ }^{9}$

Anxiety and depression is not uncommon for PPM patients. Several factors may trigger this emotional burden such as fear of device malfunction or dependency on health professionals, necessary modifications in lifestyle, limitations in daily activities, physical discomfort or technical issues including battery depletion. ${ }^{1,2,11}$ Additionally, anxiety and depression contribute to low treatment adherence, lead to unhealthy life style ${ }^{1,2}$ and provoke feelings of lack of control of the management of the disease ${ }^{11}$. Also may cause physiological changes in platelet function or blood coagulation and autonomic disbalance, thus deteriorating the already established cardiac disease. ${ }^{11}$ Last but not least, though patients perceive implantation as an extension of their life, ${ }^{11}$ the life-saving device demands psychosocial adaptation. ${ }^{2}$

Therefore, this psychiatric disturbance exerts a negative influence on patients' QoL. Deeper insights are needed to comprehensively elucidate the impact of anxiety and depression on QoL of PPM patients.

\section{AIM}

Hence, the aim of this cross-sectional study was to explore the impact of anxiety and depression on QoL of PPM recipients, as well as the demographic characteristics associated with QoL.

\section{MATERIALS AND METHODS}

\section{Study population}

In the present study, we enrolled 150 outpatients with a PPM.

The present study was cross-sectional and the method of sampling was a convenience one. Data were collected in the outpatient department of a public hospital that patients visited for scheduled follow-up.

Criteria for inclusion in the study were: a) implantation of a pacemaker, b) the ability to write and read the Greek language fluently, and c) adequate follow-up.

The excluded patients were the ones: a) who did not understand the scale sequence, b) who were speech, hearing or mentally impaired, and c) who had an additive disease or did not wish to participate in the study.

\section{Ethical considerations}

Written informed consents for participation were obtained from all patients after providing explanation about the study purpose. Participation was on a voluntary basis and anonymity was preserved. Furthermore, all participants were informed of their rights to refuse or to discontinue their participation, according to the ethical standards of the Helsinki Declaration of 1983.

\section{Procedure}

The PPM patients that agreed to participate in the study were invited to a private room to guarantee their privacy. The process of filling out the questionnaires lasted between 15 and $30 \mathrm{~min}$ and took place after patients had completed their follow-up in the outpatient clinic.

\section{Data collection}

Data were collected by completion of the following scales: I) The Hospital Anxiety and Depression Scale (HADS), and II) The Short Form (36) Health Survey (SF-36). Data for each patient also included demographic characteristics.

\section{Measurement of anxiety - depression}

To evaluate depression and anxiety, we used "The Hospital Anxiety and Depression Scale (HADS)" which was proposed in 1983 by Zigmond AS and Snaith RP. ${ }^{12}$ The scale consists of 14 questions that evaluate how patients felt during the previous week. Patients were able to answer every question in a 4-point Likert scale from 0-3. Seven out of 14 questions evaluate the level of depression and the rest seven - the anxiety level. Scores attributed to questions are summed separately for anxiety and depression, leading to two scores with a range of 0 to 21 . Higher scores indicate higher levels of anxiety and depression. ${ }^{12}$ HADs has been tested for its validity and reliability by Michopoulos et al. ${ }^{13}$

\section{Measurement of QoL}

The "SF-36 Health Survey (SF-36)" scale was used to assess patients' QoL. The SF-36 assesses physical and mental health of QoL. In detail, it consists of 36 questions comprising 8 dimensions: physical functioning, role-physical, emotional role, energy/fatigue, emotional well being, social functioning, physical pain, and general health. 
Respondents answer the questions on Likert-type scales. The scores assigned to the questions are summed up separately for the questions that evaluate the 8 dimensions. Higher scores indicate better QoL. ${ }^{14}$

\section{Statistical analysis}

Categorical data are presented with absolute and relative frequencies (\%), while continuous data are presented with median and interquartile range since they did not follow the normal distribution (tested with Kolmogorov-Smirnov criterion and graphically with Q-Qplots and Histograms). Non-parametric Mann-Whitney and Kruskal-Wallis tests were used to test for the association between patients' QoL and characteristics as well as their anxiety and depression. In addition, multiple linear regression analysis was performed to estimate the effect of anxiety/depression and patient's characteristics on their QoL. Results are presented as $\beta$ regression coefficients and $95 \%$ confidence interval ( $95 \%$ CI). The observed level of $5 \%$ was considered statistically significant. All statistical analyses were performed with SPSS version 22 (SPSS Inc, Chicago, IL, USA).

\section{RESULTS}

\section{Sample description}

According to the descriptive analysis, men accounted for the $68.7 \%$ of participants. Also, $70 \%$ of the samples were over 60 years of age, $72.7 \%$ were married, and $22.7 \%$ had primary education. The majority of the sample $(60.7 \%)$ were retired, were living in Attica (58\%), and had two children (52.7\%) (Table 1).

\section{Levels of anxiety/depression}

Results revealed that $25.3 \%$ of participants had anxiety $(n=38)$ (score $>8$ ) while $26 \%$ had depression $(n=39)$ (score $>8$ ). Cronbach's alpha coefficients were above 0.7 indicating high reliability.

\section{Levels of patients' QoL}

Table 2 presents results regarding QoL. Patients showed moderate to high levels of QoL in social functionality, energy/fatigue, emotional well-being, and physical pain (median: $50,60,72$, and 67.5 , respectively) while they showed poor levels in physical functioning, role physical and role emotional (medians: 22, 0 and 0, respectively). In addition, patients had moderate levels of general health (median 48.5).

\section{Factors affecting patient's QoL}

Tables 3-5 presents the association between patient's characteristics and QoL. Regarding physical functioning (Table 3), it was not found to be statistically significantly associated with any characteristic. Regarding role physical (Table 3), it was statistically significantly associated with age and number of children. Specifically, patients younger than 50 years old had better role physical than older patients and

Table 1. Sample description $(n=150)$

\begin{tabular}{llll}
\hline & $\mathbf{n}(\mathbf{\%})$ & & $\mathbf{n}(\mathbf{\%})$ \\
\hline Gender & & Job & \\
Male & $103(68.7 \%)$ & Unemployed & $3(2.0 \%)$ \\
Female & $47(31.3 \%)$ & Public servant & $12(8.0 \%)$ \\
\hline Age (years) & & Private employee & $15(10.0 \%)$ \\
$30-40$ & $5(3.3 \%)$ & Freelancer & $12(8.0 \%)$ \\
$41-50$ & $15(10.0 \%)$ & Household & $17(11.3 \%)$ \\
$51-60$ & $25(16.7 \%)$ & Pensioner & $91(60.7 \%)$ \\
$61-70$ & $68(45.3 \%)$ & Residency & \\
$71-80$ & $37(24.7 \%)$ & Attica & $87(58.0 \%)$ \\
Family Status & & County capital & $29(19.3 \%)$ \\
Married & $109(72.7 \%)$ & Small city & $13(8.7 \%)$ \\
Single & $16(10.7 \%)$ & Rural area & $21(14.0 \%)$ \\
Divorced & No of children & $16(10.7 \%)$ \\
Widowed & $11(7.3 \%)$ & None & $31(20.7 \%)$ \\
Living together & $12(8.0 \%)$ & One & $79(52.7 \%)$ \\
\hline Education & $2(1.3 \%)$ & Two & $24(16.0 \%)$ \\
Primary & $34(22.7 \%)$ & More than two & \\
Secondary & $58(38.7 \%)$ & & \\
University & $53(35.3 \%)$ & & \\
MSc PhD & $5(3.3 \%)$ & & \\
\hline
\end{tabular}


Table 2. Levels of patient's QoL $(\mathrm{N}=150)$

\begin{tabular}{ll}
\hline & Median (IQR) \\
\hline Physical Functioning (range: 0-100) & $22(16-27)$ \\
Role physical (range: 0-100) & $0(0-75)$ \\
Role emotional (range: $0-100)$ & $0(0-100)$ \\
Energy/fatigue (range: 0-100) & $60(45-70)$ \\
Emotional well-being (range: $0-100)$ & $72(52-84)$ \\
Social functioning (range: 0-100) & $50(50-87.5)$ \\
Pain (range: 0-100) & $67.5(45-90)$ \\
General health (range: 0-100) & $48.5(35-65)$ \\
\hline
\end{tabular}

IQR: Interquartile range those with two or more children. Regarding role emotional (Table 3), it was statistically significantly associated with age and education. Specifically, patients younger than 50 years old had better role emotional than older patients. Similarly, patients with secondary education had better emotional role.

Regarding energy/fatigue (Table 4), it was not found to be statistically significantly associated with any characteristic. Regarding emotional well-being (Table 4), it was not found to be statistically significantly associated with any characteristic. Regarding social functioning (Table 4), it was statistically significantly associated with age and family status. Specifically, patients younger than 50 years old and

Table 3. Association between patient's characteristics and QoL in dimensions: physical functioning, role physical, role emotional

\begin{tabular}{|c|c|c|c|c|c|c|}
\hline & \multicolumn{2}{|c|}{ Physical functioning } & \multicolumn{2}{|l|}{ Role physical } & \multicolumn{2}{|c|}{ Role emotional } \\
\hline & $\begin{array}{l}\text { Median } \\
\text { (IQR) }\end{array}$ & $p$ & $\begin{array}{l}\text { Median } \\
\text { (IQR) }\end{array}$ & $p$ & $\begin{array}{l}\text { Median } \\
\text { (IQR) }\end{array}$ & $p$ \\
\hline Gender & & 0.382 & & 0.576 & & 0.405 \\
\hline Male & $21(11-26)$ & & $0(0-75)$ & & $0(0-100)$ & \\
\hline Female & $22(10-30)$ & & $0(0-100)$ & & $0(0-100)$ & \\
\hline Age, years & & 0.135 & & 0.025 & & 0.005 \\
\hline$\leq 50$ & $25.5(16-27.5)$ & & $87.5(0-100)$ & & $100(50-100)$ & \\
\hline $51-60$ & $26(17-27)$ & & $0(0-50)$ & & $0(0-67)$ & \\
\hline $61-70$ & $21(11-26)$ & & $0(0-87.5)$ & & $0(0-100)$ & \\
\hline $71-80$ & $20(6-28)$ & & $0(0-50)$ & & $0(0-33)$ & \\
\hline Family Status & & 0.283 & & 0.309 & & 0.491 \\
\hline Married / Living together & $21(11-27)$ & & $0(0-100)$ & & $0(0-100)$ & \\
\hline Single & $23.5(21-26.5)$ & & $0(0-100)$ & & $0(0-100)$ & \\
\hline Divorced / Widowed & $22(15-27)$ & & $0(0-50)$ & & $0(0-67)$ & \\
\hline Education & & 0.827 & & 0.060 & & 0.011 \\
\hline Primary & $20(14-28)$ & & $0(0-50)$ & & $0(0-33)$ & \\
\hline Secondary & $21(15-26)$ & & $37.5(0-100)$ & & $50(0-100)$ & \\
\hline University & $21.5(10-28)$ & & $0(0-75)$ & & $0(0-100)$ & \\
\hline Job & & 0.728 & & 0.441 & & 0.565 \\
\hline Employee & $20(10-26)$ & & $0(0-100)$ & & $33(0-100)$ & \\
\hline Household / Unemployed & $22.5(15-28.5)$ & & $0(0-75)$ & & $0(0-100)$ & \\
\hline Pensioner & $21(14-27)$ & & $0(0-75)$ & & $0(0-100)$ & \\
\hline Residency & & 0.679 & & 0.076 & & 0.309 \\
\hline Attica & $21(10-26)$ & & $25(0-100)$ & & $0(0-100)$ & \\
\hline County capital & $23(15-28)$ & & $0(0-75)$ & & $0(0-67)$ & \\
\hline Small city / Rural area & $19(15-27)$ & & $0(0-25)$ & & $0(0-67)$ & \\
\hline No of children & & 0.531 & & 0.024 & & 0.145 \\
\hline None & $23.5(21-26)$ & & $0(0-100)$ & & $0(0-100)$ & \\
\hline One & $20(15-28)$ & & $0(0-0)$ & & $0(0-33)$ & \\
\hline Two & $21(10-27)$ & & $25(0-100)$ & & $33(0-100)$ & \\
\hline More than two & $21.5(14-26)$ & & $25(0-75)$ & & $0(0-100)$ & \\
\hline
\end{tabular}


the married ones had better social functioning.

Regarding pain (Table 5), it was found to be statistically significantly associated with age. More specifically, patients aged under 50 had better QoL. Regarding general health (Table 5), it was statistically significantly associated with residency. Specifically, patients living in Attica had better general health.

\section{Association between anxiety/depression and QoL}

Table 6 presents the association between patient's anxiety/ depression and QoL on a univariate level. All subscales of QoL were significantly associated with anxiety of patients $(p<0.05)$.
Patients with anxiety had lower scores, thus meaning worse QoL in all subscales. Similarly, depression was significantly associated with QoL apart from physical functioning and role physical. Patients with depression had lower scores thus meaning worse QoL in the remaining subscales.

\section{Effect of characteristics and anxiety/ depression on QoL}

Multiple linear regression analysis was performed with dependent variables the subscales of patient's QoL in order to estimate the effect of patient characteristics and their anxiety/depression (independent factors). Tables 7-9 show that after controlling for patient's characteristics, anxiety did

Table 4. Association between patient's characteristics and QoL in dimensions: energy/fatigue, emotional well-being, social functioning

\begin{tabular}{|c|c|c|c|c|c|c|}
\hline & \multicolumn{2}{|l|}{ Energy/Fatigue } & \multicolumn{2}{|c|}{ Emotional well-Being } & \multicolumn{2}{|c|}{ Social Functioning } \\
\hline & $\begin{array}{l}\text { Median } \\
\text { (IQR) }\end{array}$ & $p$ & $\begin{array}{l}\text { Median } \\
\text { (IQR) }\end{array}$ & $p$ & $\begin{array}{l}\text { Median } \\
\text { (IQR) }\end{array}$ & $p$ \\
\hline Gender & & 0.283 & & 0.091 & & 0.523 \\
\hline Male & $60(35-70)$ & & $68(48-84)$ & & $50(37.5-100)$ & \\
\hline Female & $55(35-65)$ & & $64(36-76)$ & & $50(37.5-87.5)$ & \\
\hline Age & & 0.287 & & 0.724 & & 0.033 \\
\hline$\leq 50$ & $60(47.5-70)$ & & $70(52-80)$ & & $87.5(68.75-100)$ & \\
\hline $51-60$ & $60(45-75)$ & & $68(64-76)$ & & $50(37.5-75)$ & \\
\hline $61-70$ & $60(35-70)$ & & $64(44-76)$ & & $50(31.25-100)$ & \\
\hline $71-80$ & $50(30-65)$ & & $68(40-84)$ & & $50(25-75)$ & \\
\hline Family Status & & 0.219 & & 0.743 & & 0.008 \\
\hline Married / Living together & $60(45-70)$ & & $68(48-80)$ & & $62.5(50-100)$ & \\
\hline Single & $60(32.5-72.5)$ & & $68(42-78)$ & & $62.5(18.75-87.5)$ & \\
\hline Divorced / Widowed & $55(25-65)$ & & $60(40-84)$ & & $50(12.5-75)$ & \\
\hline Education & & 0.067 & & 0.055 & & 0.523 \\
\hline Primary & $50(20-65)$ & & $56(40-76)$ & & $50(37.5-75)$ & \\
\hline Secondary & $60(40-70)$ & & $66(40-84)$ & & $75(25-100)$ & \\
\hline University & $60(50-70)$ & & $68(52-84)$ & & $50(37.5-87.5)$ & \\
\hline Job & & 0.065 & & 0.320 & & 0.831 \\
\hline Employee & $65(45-75)$ & & $68(56-80)$ & & $75(37.5-87.5)$ & \\
\hline Household / Unemployed & $52.5(37.5-62.5)$ & & $64(44-78)$ & & $75(50-75)$ & \\
\hline Pensioner & $55(30-70)$ & & $64(40-80)$ & & $50(25-100)$ & \\
\hline Residency & & 0.765 & & 0.477 & & 0.220 \\
\hline Attica & $60(45-70)$ & & $68(48-84)$ & & $75(37.5-100)$ & \\
\hline County capital & $60(30-70)$ & & $64(40-76)$ & & $50(25-75)$ & \\
\hline Small city / Rural area & $57.5(30-70)$ & & $64(44-80)$ & & $50(25-87.5)$ & \\
\hline No of children & & 0.345 & & 0.872 & & 0.584 \\
\hline None & $70(40-80)$ & & $68(42-78)$ & & $50(18.75-93.75)$ & \\
\hline One & $55(30-65)$ & & $68(40-84)$ & & $50(25-87.5)$ & \\
\hline Two & $60(40-70)$ & & $64(48-84)$ & & $50(37.5-100)$ & \\
\hline More than two & $55(40-67.5)$ & & $72(56-76)$ & & $68.75(50-100)$ & \\
\hline
\end{tabular}


Table 5. Association between patient's characteristics and QoL in pain and general health dimensions

\begin{tabular}{|c|c|c|c|c|}
\hline & \multicolumn{2}{|l|}{ Pain } & \multicolumn{2}{|c|}{ General Health } \\
\hline & $\begin{array}{l}\text { Median } \\
\text { (IQR) }\end{array}$ & $p$ & $\begin{array}{l}\text { Median } \\
\text { (IQR) }\end{array}$ & $p$ \\
\hline Gender & & 0.882 & & 0.648 \\
\hline Male & $67.5(32$ & & $50(32-65)$ & \\
\hline Female & $67.5(32$. & & $50(30-65)$ & \\
\hline Age, years & & 0.018 & & 0.514 \\
\hline$\leq 50$ & $100(67.5$ & & $51(28.5-70)$ & \\
\hline $51-60$ & $57.5(45-$ & & $45(40-65)$ & \\
\hline $61-70$ & $67.5(32$ & & $55(35-65)$ & \\
\hline $71-80$ & $45(22.5-$ & & $45(20-62)$ & \\
\hline Family Status & & 0.137 & & 0.343 \\
\hline Married / Living together & $67.5(32$. & & $50(30-65)$ & \\
\hline Single & $67.5(45-$ & & $55(45-67.5)$ & \\
\hline Divorced / Widowed & $45(22.5-$ & & $42(20-55)$ & \\
\hline Education & & 0.346 & & 0.324 \\
\hline Primary & $57.5(35-$ & & $42.5(27-60)$ & \\
\hline Secondary & $57.5(32$ & & $55(35-65)$ & \\
\hline University & $77.5(45-$ & & $50(35-70)$ & \\
\hline Job & & 0.596 & & 0.179 \\
\hline Employee & $67.5(45-$ & & $55(40-70)$ & \\
\hline Household / Unemployed & $67.5(40-$ & & $42.5(30-55)$ & \\
\hline Pensioner & $67.5(32$. & & $50(30-65)$ & \\
\hline Residency & & 0.367 & & 0.006 \\
\hline Attica & $77.5(35-$ & & $55(35-70)$ & \\
\hline County capital & $57.5(32$. & & $40(27-45)$ & \\
\hline Small city / Rural area & $57.5(32$. & & $51(30-65)$ & \\
\hline No of children & & 0.455 & & 0.484 \\
\hline None & $67.5(50-$ & & $53.5(45-67.5)$ & \\
\hline One & $57.5(32$. & & $45(30-55)$ & \\
\hline Two & $67.5(32$. & & $55(30-70)$ & \\
\hline More than two & $57.5(22$. & & $42.5(32.5-65)$ & \\
\hline
\end{tabular}

Table 6. Association between patient's anxiety/depression and QoL

\begin{tabular}{lllllll}
\hline & Anxiety & & \multicolumn{3}{l}{ Depression } \\
\cline { 2 - 7 } & $\begin{array}{l}\text { No } \\
\text { Median (IQR) }\end{array}$ & $\begin{array}{l}\text { Yes } \\
\text { Median (IQR) }\end{array}$ & $\boldsymbol{p}$ & $\begin{array}{l}\text { No } \\
\text { Median (IQR) }\end{array}$ & $\begin{array}{l}\text { Yes } \\
\text { Median (IQR) }\end{array}$ & $\boldsymbol{p}$ \\
\hline Physical functioning & $22.5(15.5-28)$ & $13(5-21)$ & 0.001 & $21(14-27)$ & $21(5-26)$ & 0.474 \\
Role physical & $12.5(0-100)$ & $0(0-50)$ & 0.045 & $0(0-100)$ & $0(0-50)$ & 0.060 \\
Role emotional & $17(0-100)$ & $0(0-33)$ & 0.043 & $33(0-100)$ & $0(0-33)$ & 0.037 \\
Energy/fatigue & $60(50-72.5)$ & $37.5(20-60)$ & 0.001 & $60(50-75)$ & $35(20-55)$ & 0.001 \\
Emotional well-being & $72(56-84)$ & $46(36-64)$ & 0.001 & $72(56-84)$ & $40(32-64)$ & 0.001 \\
Social functioning & $75(50-100)$ & $25(12.5-50)$ & 0.001 & $75(50-100)$ & $25(12.5-50)$ & 0.001 \\
Pain & $70(45-100)$ & $27.5(10-77.5)$ & 0.001 & $70(45-100)$ & $45(12.5-77.5)$ & 0.001 \\
General health & $50(35-70)$ & $45(15-55)$ & 0.007 & $55(40-70)$ & $30(15-55)$ & 0.001 \\
\hline
\end{tabular}


Table 7. Impact of patient's characteristics and anxiety/depression on QoL in dimensions of physical functioning, role physical, and role emotional

\begin{tabular}{|c|c|c|c|c|c|c|}
\hline & \multicolumn{2}{|c|}{ Physical functioning } & \multicolumn{2}{|l|}{ Role physical } & \multicolumn{2}{|l|}{ Role emotional } \\
\hline & $\begin{array}{l}\beta \text { coeff } \\
(95 \% \mathrm{CI})\end{array}$ & $p$ & $\begin{array}{l}\beta \text { coeff } \\
(95 \% \mathrm{CI})\end{array}$ & $p$ & $\begin{array}{l}\beta \text { coeff } \\
(95 \% \mathrm{CI})\end{array}$ & $p$ \\
\hline \multicolumn{7}{|l|}{ Age } \\
\hline$\leq 50$ & - & & Reference & & Reference & \\
\hline $51-60$ & - & & $-22.8(-47.3,1.7)$ & 0.068 & $-32.5(-58.1,-6.9)$ & 0.013 \\
\hline $61-70$ & - & & $-26.6(-46.7,-6.5)$ & 0.010 & $-25.9(-46.8,-5.0)$ & 0.015 \\
\hline $71-80$ & - & & $-34.4(-56.9,-12.0)$ & 0.003 & $-37.6(-60.5,-14.8)$ & 0.001 \\
\hline \multicolumn{7}{|l|}{ Education } \\
\hline Primary & - & & - & & Reference & \\
\hline Secondary & - & & - & & $25.4(7.3,43.5)$ & 0.006 \\
\hline University & - & & - & & $14.4(-3.8,32.6)$ & 0.120 \\
\hline \multicolumn{7}{|l|}{ No of children } \\
\hline None & - & & Reference & & - & \\
\hline One & - & & $-8.0(-32.9,16.8)$ & 0.523 & - & \\
\hline Two & - & & $16.0(-5.9,38.0)$ & 0.151 & - & \\
\hline More than two & - & & $19.5(-6.5,45.5)$ & 0.140 & - & \\
\hline \multicolumn{7}{|l|}{ Anxiety } \\
\hline No & Reference & & Reference & & Reference & \\
\hline Yes & $-8.2(-12.3,-4.1)$ & 0.001 & $-10.3(-26.4,5.7)$ & 0.206 & $-14.4(-34.2,5.3)$ & 0.151 \\
\hline \multicolumn{7}{|l|}{ Depression } \\
\hline No & - & & - & & Reference & \\
\hline Yes & - & & - & & $-5.1(-24.5,14 ., 3)$ & 0.604 \\
\hline
\end{tabular}

not affect role physical, role emotional, energy/fatigue, and general health (confounding effect). Similarly, depression did not affect role emotional and pain.

Regarding the rest subscales, patients with anxiety had 8.2 points worse physical functioning (95\% CI: $-12.3,-4.1$, $p=0.001), 10.4$ points worse emotional well-being $(95 \%$ CI: $-17.7,-3.1, p=0.006), 17$ points worse social functioning (95\% CI: $-28.4,-5.6, p=0.004)$ and 16.9 points worse pain (95\% CI: $-30.4,-3.4, p=0.015)$. Similarly patients with depression had 22.8 points worse energy/fatigue (95\% CI: $-31.2,-14.4, p=0.001), 15$ points worse emotional well-being (95\% CI: $-22.3,-7.8, p=0.001), 15$ points worse social functioning (95\% CI: $-26.5,-3.5, p=0.011$ ), and 9.6 points worse general health ( $95 \% \mathrm{CI}:-17.7,-1.6, p=0.019)$.

\section{DISCUSSION}

According to the results of the present study, patients showed moderate to high levels of QoL in social functionality, energy/fatigue, emotional well-being, and pain whereas poor levels in physical functioning, role physical and emotional. In addition, patients had moderate levels of general health. Barros et al., ${ }^{7}$ who explored 107 clinically stable patients (aged 69.3 \pm 12.6 , 49.5\% female) with an implantation time span of 3 up to 12 months showed the lowest
SF-36 scores in physical aspects, and the highest ones in social aspects. Similarly, a prior study conducted by Oliveira et al., ${ }^{15}$ who explored 139 clinically stable recipients (aged $59 \pm 14,60.4 \%$ female) indicated the decline in functional class as the strongest determinant of low QoL. Moreover, improvements in all SF-36 domains, were observed at 3 and 12 months among 720 recipients of leadless device (aged $76 \pm 11$ years, $41 \%$ female). ${ }^{16} \mathrm{~A}$ relevant research demonstrated QoL improvements in $75 \%$ of recipients but $40 \%$ expressed worries about the device. ${ }^{17}$

Noteworthy, a great effort is noticed globally to improve all domains in QoL through incorporating measurements pre- and post-implantation in order to evaluate effectiveness of therapy. Furthermore, scheduled follow-up (faceto-face visits) in cardiology departments is prerequisite to maintain QoL and preferred by the majority of patients $(83 \%) .{ }^{17}$ Meanwhile, the alternative method of remote monitoring may increase QoL six months post implantation. ${ }^{18}$ In contemporary times, remote follow-up among PPM patients is a cost-effective option which doesn't demand attendance to cardiology services. From patients' perspective, communicating with clinicians from home is acknowledged as a safe and convenient approach, thus indirectly improving QoL. ${ }^{19,20}$

Results revealed that almost a quarter of participants had anxiety (25.3\%) and depression (26.0\%). A relevant 
Table 8. Impact of patient's characteristics and anxiety/depression on QoL in dimensions of energy/fatigue, emotional well-being, and social functioning

\begin{tabular}{|c|c|c|c|c|c|c|}
\hline & \multicolumn{2}{|l|}{ Energy/Fatigue } & \multicolumn{2}{|c|}{ Emotional well-being } & \multicolumn{2}{|l|}{ Social functioning } \\
\hline & $\begin{array}{l}\beta \text { coeff } \\
(95 \% \mathrm{CI})\end{array}$ & $p$ & $\begin{array}{l}\beta \text { coeff } \\
(95 \% \mathrm{CI})\end{array}$ & $p$ & $\begin{array}{l}\beta \text { coeff } \\
(95 \% \mathrm{CI})\end{array}$ & $p$ \\
\hline \multicolumn{7}{|l|}{ Age, years } \\
\hline$\leq 50$ & - & & - & & Reference & \\
\hline $51-60$ & - & & - & & $-18.3(-34.6,-1.9)$ & 0.029 \\
\hline $61-70$ & - & & - & & $-15.7(-28.4,-3.0)$ & 0.016 \\
\hline $71-80$ & - & & - & & $-25.0(-39.3,-10.7)$ & 0.001 \\
\hline \multicolumn{7}{|l|}{ Family Status } \\
\hline Married / Living together & - & & - & & Reference & \\
\hline Single & - & & - & & $-8.3(-21.8,5.2)$ & 0.225 \\
\hline Divorced / Widowed & - & & - & & $-2.5(-14.3,9.4)$ & 0.682 \\
\hline \multicolumn{7}{|l|}{ Anxiety } \\
\hline No & Reference & & Reference & & Reference & \\
\hline Yes & $-4.1(-12.4,4.2)$ & 0.328 & $-10.4(-17.7,-3.1)$ & 0.006 & $-17.0(-28.4,-5.6)$ & 0.004 \\
\hline \multicolumn{7}{|l|}{ Depression } \\
\hline No & Reference & & Reference & & Reference & \\
\hline Yes & $-22.8(-31.2,-14.4)$ & 0.001 & $-15.0(-22.3,-7.8)$ & 0.001 & $-15.0(-26.5,-3.5)$ & 0.011 \\
\hline
\end{tabular}

Table 9. Impact of patient's characteristics and anxiety/depression on QoL in dimensions of pain and general health

\begin{tabular}{|c|c|c|c|c|}
\hline & \multicolumn{2}{|l|}{ Pain } & \multicolumn{2}{|l|}{ General Health } \\
\hline & $\begin{array}{l}\beta \text { coeff } \\
(95 \% \mathrm{CI})\end{array}$ & $p$ & $\begin{array}{l}\beta \text { coeff } \\
(95 \% \mathrm{CI})\end{array}$ & $p$ \\
\hline \multicolumn{5}{|l|}{ Age, years } \\
\hline$\leq 50$ & Reference & & - & \\
\hline $51-60$ & $-9.1(-28.7,10.6)$ & 0.364 & - & \\
\hline $61-70$ & $-11.3(-26.3,3.7)$ & 0.138 & - & \\
\hline $71-80$ & $-21.0(-37.3,-4.6)$ & 0.012 & - & \\
\hline \multicolumn{5}{|l|}{ Residency } \\
\hline Attica & - & & Reference & \\
\hline County capital & - & & $-7.7(-16.9,1.6)$ & 0.103 \\
\hline Small city / Rural area & - & & $-2.9(-11.2,5.4)$ & 0.490 \\
\hline \multicolumn{5}{|l|}{ Anxiety } \\
\hline No & Reference & & Reference & \\
\hline Yes & $-16.9(-30.4,-3.4)$ & 0.015 & $-4.0(-13.8,5.7)$ & 0.413 \\
\hline \multicolumn{5}{|l|}{ Depression } \\
\hline No & Reference & & Reference & \\
\hline Yes & $-2.5(-16.1,11.1)$ & 0.720 & $-9.6(-17.7,-1.6)$ & 0.019 \\
\hline
\end{tabular}

recent study among 250 PPM recipients (median 71 years) showed that $27.2 \%$ and $14 \%$ experienced high levels of anxiety and depression, respectively. The same researchers also showed that $69.6 \%$ of participants described themselves as anxious while $19.6 \%$ and $23.2 \%$ reported that they experienced severe anxiety about device functioning and heart rate disorder, respectively. ${ }^{2}$ Psychiatric disturbance does not seem to be a recent issue in pacing therapy. A prior study showed that $19.1 \%(n=16)$ among 84 recipients had a psychiatric diagnosis. The most frequent diagnoses were adjustment disorder (5.9\%) and major depressive episode $(4.7 \%) .^{21}$ 
At the other end of the spectrum, implantation is a challenge for patients with mental illness. Depression is not an absolute contraindication to permanent cardiac pacing. Furthermore, the implanted device has beneficial effects on chronic bradycardia, which is associated with depression but follow-up and monitoring are needed. Strikingly, suicide attempts by intentionally removing the pacemaker system are rare, but they have adverse clinical outcomes. ${ }^{22}$

Apart from physical functioning and role physical in depression, all remaining QoL subscales were associated with anxiety and depression. The potential influence of anxiety and depression on QoL is a notable aspect in the field of PPM. Interestingly, psychiatric comorbidity exerts a negative influence on recovery or elevates the risk of future cardiovascular events, which in turn affect QoL. A three month cardiac rehabilitation program significantly improves the triad: anxiety, depression, QoL in patients with various cardiac pathology, including implantation. ${ }^{23}$

Another possible explanation for low QoL among PPM recipients who experience psychiatric disturbance is through indirect pathways, such as fatigue. For example, recipients with high levels of anxiety and depression feel more fatigue, ${ }^{2}$ while those who don't experience QoL improvement after implantation, also report high levels of fatigue ${ }^{1}$.

Therefore, QoL measurement is beneficial when including both cardiovascular and psychological assessment. This approach helps clinicians who specialize in the field of cardiology to gain deeper understanding of the patients' needs. ${ }^{10,24,25}$ QoL before implantation is significantly lower compared to the general population. ${ }^{26}$ Thus health care professionals are aware to take care of an already "vulnerable" population in terms of QoL.

In terms of demographic characteristics, results showed better QoL in physical and emotional role, social functioning, and in pain among participants younger than 50 years old. Possibly, the concept of QoL in younger age is more closely related to autonomy and independence than to the presence of disease. Minimization of the interval between the time of diagnosis (indication for pacing therapy) and implantation procedure might be a predictor for better QoL. ${ }^{27}$

However, as the age is advancing, the QoL is worsening in terms of functional capacity. Notably, QoL measurement among the elderly is not always attainable due to their diminished life expectancy and comorbidities. ${ }^{7}$ Interestingly, age as a non-modifiable factor, which is inversely related to QoL, must be considered when implementing individualized interventions. ${ }^{7}$ However, patients in a waiting list for implantation are usually older than other comorbidity such as epilepsy or diabetes mellitus. ${ }^{26}$

Regarding the other demographic characteristics related to QoL, results showed better physical role among patients having two or more children and better social functioning in married patients. This encouraging finding is largely attributed to support within family which is an already known area among cardiac patients. ${ }^{27-29}$ Contrariwise, patients without a partner display low QoL in the SF-36 mental component. ${ }^{15}$ Family members, especially spouses, are the first to notice worsening of symptoms and generally provide a context of support in several daily aspects or practical issues. Also, family members may share common cultural background, which means appreciation of patients' deeper perceptions while adherence to treatment is higher in patients from cohesive families. ${ }^{29}$ Notably, the more the support, the less the anxiety among cardiac patients. ${ }^{30}$

\section{Study Limitations}

Some limitations of the study must be acknowledged. First, convenience sampling is one of the limitations because this method is not representative of all PPM patients in Greece. Furthermore, there was no longitudinal design with follow-up data on the same patients that might permit evaluation of possible changes in all dimensions (anxiety, depression and QoL). It would be interesting to monitor anxiety, depression, and QoL before implantation and 12 or 24 months after baseline. The sample size was relatively small although significant associations were observed. Future research might involve a larger sample size. Self-report questionnaires are not considered precise to make a diagnosis of anxiety, depression but they are only additive to psychiatric evaluation. The strengths of the study include the use of widespread instruments, which may permit comparisons among cardiac populations across the world.

\section{CONCLUSIONS}

According to the present results, almost a quarter of patients experienced anxiety and depression while poor QoL was found in physical functioning, role physical and emotional. Additionally, anxiety and depression were associated with QoL.

These results demonstrate the necessity to identify and treat the psychiatric disturbance and are also raising the issue of routine psychiatric evaluation before and after pacemaker implantation.

In clinical practice, QoL measurement can be useful for monitoring progress, and measuring clinical outcomes.

From a practical perspective, the present knowledge may help clinicians to provide a better and research-based care for this steadily increasing population.

Having as ultimate goal to improve QoL, then further studies need to research more on anxiety and depression and to examine ways to minimize this burden using a longitudinal design whereby patients are followed.

\section{Funding}

This research received no specific grant from any funding agency in the public, commercial or not-for-profit sectors. 


\section{Conflict of Interest}

The author declares no conflict of interest.

\section{Ethical approval}

The study was approved by the Medical Research Ethics Committee of the hospital.

\section{Research involving human participants}

All procedures performed in the studies involving human participants were in accordance with the ethical standards of the institutional and/or national research committee and with the 1964 Helsinki declaration and its later amendments or comparable ethical standards.

\section{REFERENCES}

1. Polikandrioti M, Tzirogiannis K, Zyga S, et al. Assessment of fatigue in patients with a permanent cardiac pacemaker: prevalence and associated factors. Arch Med Sci Atheroscler Dis 2018; 3:e166-e173.

2. Polikandrioti M, Tzirogiannis K, Zyga S, et al. Effect of anxiety and depression on the fatigue of patients with a permanent pacemaker. Arch Med Sci Atheroscler Dis 2018; 3:e8-e17.

3. Sideris S, Archontakis S, Dilaveris P, et al. Leadless cardiac pacemakers: current status of a modern approach in pacing. Hellenic J Cardiol 2017; 58(6):403-10.

4. Bhatia N, El-Chami M. Leadless pacemakers: a contemporary review. J Geriatr Cardiol 2018; 15(4):249-53.

5. Bernard ML. Pacing without wires: leadless cardiac pacing. Ochsner J 2016; 16(3):238-42.

6. Bradshaw PJ, Stobie P, Knuiman MW, et al. Trends in the incidence and prevalence of cardiac pacemaker insertions in an ageing population. Open Heart 2014; 1(1):e000177.

7. Barros RT, Carvalho SM, Silva MA, et al. Evaluation of patients' quality of life aspects after cardiac pacemaker implantation. Rev Bras Cir Cardiovasc 2014; 29(1):37-44.

8. Udo EO, van Hemel NM, Zuithoff NP, et al. Long term quality-of-life in patients with bradycardia pacemaker implantation. Int J Cardiol 2013; 168(3):2159-63.

9. Lopez-Villegas A, Catalan-Matamoros D, Lopez-Liria R, et al. Healthrelated quality of life on tele-monitoring for users with pacemakers 6 months after implant: the NORDLAND study, a randomized trial. BMC Geriatr 2018; 18(1):223.

10. Hand C. Measuring health-related quality of life in adults with chronic conditions in primary care settings: Critical review of concepts and 3 tools. Can Fam Physician 2016; 62(7):e375-83.

11. Figueroa C, Alcocer L, Ramos B. Psychological intervention to modify anxiety, depression and quality of life in patients with an implantable pacemaker. Psychology 2016; 7:374-81.

12. Zigmond AS, Snaith RP. The hospital anxiety and depression scale. Acta Psychiatr Scand 1983; 67:361-70.
13. Michopoulos I, Douzenis A, Kalkavoura C, et al. Hospital anxiety and depression scale (HADs): validation in a Greek general hospital sample. Ann Gen Psychiatry 2008; 7:4.

14. Ware JE. SF-36 Health Survey update. Spine 2000; 25(24):3130-9.

15. Oliveira BG, Velasquez-Melendez G, Rincón LG, et al. Health-related quality of life in Brazilian pacemaker patients. Pacing Clin Electrophysiol 2008; 31(9):1178-83.

16. Tjong FVY, Beurskens NEG, de Groot JR, et al. Health-related quality of life impact of a transcatheter pacing system. J Cardiovasc Electrophysiol 2018; 29(12):1697-704.

17. Haugaa KH, Potpara TS, Boveda S, et al. Patients' knowledge and attitudes regarding living with implantable electronic devices: results of a multicentre, multinational patient survey conducted by the European Heart Rhythm Association. Europace 2018; 20(2):386-91.

18. Comoretto RI, Facchin D, Ghidina M, et al. Remote control improves quality of life in elderly pacemaker patients versus standard ambulatory-based follow-up. J Eval Clin Pract 2017; 23(4):681-9.

19. Catalan-Matamoros D, Lopez-Villegas A, Lappegård KT, et al. Assessing communication during remote follow-up of users with pacemakers in Norway: The NORDLAND study, a randomized trial. Int J Environ Res Public Health 2020; 17(20):7678.

20. Catalan-Matamoros D, Lopez-Villegas A, Tore-Lappegard K, et al. Patients' experiences of remote communication after pacemaker implant: The NORDLAND study. PLoS One 2019; 14(6):e0218521.

21. Aydemir O, Ozmen E, Küey L, et al. Psychiatric morbidity and depressive symptomatology in patients with permanent pacemakers. Pacing Clin Electrophysiol 1997; 20(6):1628-32.

22. Che X, Abdelwahed YS, Wang X, et al. Pacemaker implantation in patients with major depression, should it be of concern? A case report and literature review. BMC Cardiovasc Disord 2020;20(1):279.

23. Rosic D, Krstacic G, Krstacic A, et al. The role of ambulatory cardiac rehabilitation in improvement of quality of life, anxiety, and depression. Psychiatr Danub 2020; 32(Suppl 4):496-504.

24. Polikandrioti M, Goudevenos J, Michalis LK, et al. Correlation between the type of acute coronary syndrome with the needs of hospitalized patients. Glob J Health Sci 2015; 8(7):126-34.

25. Polikandrioti M, Panoutsopoulos G, Tsami A, et al. Assessment of quality of life and anxiety in heart failure outpatients. Arch Med Sci Atheroscler Dis 2019; 4:e38-e46.

26. Martijn Van Eck JW, Van Hemel NM, Kelder JC, et al, FOLLOWPACE investigators. Poor health-related quality of life of patients with indication for chronic cardiac pacemaker therapy. Pacing Clin Electrophysiol 2008; 31(4):480-6.

27. Rondón García LM, Ramírez Navarrro JM. The impact of quality of life on the health of older people from a multidimensional perspective. J Aging Res 2018; 2018:4086294.

28. Allemann H, Strömberg A, Thylén I. Perceived social support in persons with heart failure living with an implantable cardioverter defibrillator: a cross-sectional explorative study. J Cardiovasc Nurs 2018; 33:E1-8.

29. Polikandrioti M, Kalafatakis F, Koutelekos I, et al. Fatigue in heart failure outpatients: levels, associated factors, and the impact on quality of life. Arch Med Sci Atheroscler Dis 2019; 4:e103-e112.

30. Legaki F, Babatsikou F, Koutis C, et al. State and trait anxiety in islander cardiac patients: associated factors and the impact of perceived social support. Arch Med Sci Atheroscler Dis 2020; 5:e85-e97. 


\title{
Качество жизни пациентов с кардиостимулятором: уровни, связанные характеристики и влияние тревоги и депрессии
}

\author{
Мария Поликандриоти \\ университет Западной Аттики, Афинь, Греция
}

Адрес для корреспонденции: Мария Поликандриоти, Университет Западной Аттики, Афины, Греция; E-mail: mроlik2006@уahoo.соm

Дата получения: 20 января 2021 Дата приемки: 15 апреля 2021 Дата публикации: 28 февраля 2022

Образец цитирования: Polikandrioti M. Quality of life of patients with cardiac pacemaker: levels, associated characteristics, and the impact of anxiety and depression. Folia Med (Plovdiv) 2022;64(1):117-27. doi: 10.3897/folmed.64.e63234.

\section{Резюме}

Введение: Имплантация постоянного электрокардиостимулятора (ПЭК) улучшает качество жизни пациентов (КЖ). Однако психические расстройства могут неблагоприятно влиять на качество жизни и влиять на клинический исход.

Цель: Изучить влияние тревоги и депрессии на качество жизни реципиентов ПЭК.

Материалы и методы: Было обследовано 150 реципиентов ПЭК. Сбор данных осуществлялся путём заполнения Шкалы клинической тревоги и депрессии (HADS) и стандартизированного опросника SF-36. Демографические характеристики также были включены. Уровень статистической значимости составил $p<0.05$.

Результаты: Почти четверть пациентов испытывали тревогу (25.3\%) и депрессию (26.0\%). Что касается качества жизни, пациенты демонстрировали умеренный или высокий уровень социальной функциональности, энергии/усталости, эмоционального благополучия и боли (в среднем 50, 60, 72 и 67.5 соответственно) и низкие уровни физической функции, физической роли и эмоциональной роли (в среднем соответственно: 22,0 и 0). Кроме того, пациенты имели средние показатели общего самочувствия (в среднем 48.5). Все субшкалы КЖ были отрицательно связаны с тревогой и депрессией у пациентов на одномерном уровне $(p<0.05)$. Что касается демографических переменных, влияющих на качество жизни, возраст статистически значимо связан с физической ролью ( $p=0.025)$, эмоциональной ролью ( $p=0.005)$, социальным функционированием $(p=0.033)$ и болью ( $p=0.018)$. Кроме того, физическая роль была статистически значимо связана с количеством детей $(p=0.024)$, эмоциональная роль с уровнем образования $(p=0.011)$, социальное функционирование с семейным положением $(p=0.018)$ и общее состояние здоровья с местом жительства $(p=0.006)$.

Заключение: Демографические характеристики и тревога/депрессия связаны с качеством жизни. Лучшее понимание этих взаимосвязей может помочь клиницистам планировать рациональные и рентабельные вмешательства.

\section{Ключевые слова}

тревога, депрессия, качество жизни, HADS, SF36, кардиостимулятор 